\title{
Effect of Anticholinergic Medication on Positive and Negative Symptoms in Medication-Free Schizophrenic Patients
}

\author{
Rajiv Tandon, Nancy A. Mann, William H. Eisner, and Nancy Coppard
}

Received July 31, 1989; revised version received October 27, 1989; accepted November 9, 1989.

\begin{abstract}
It is generally assumed that anticholinergic drugs have no effects on schizophrenic symptomatology. A few studies, however, indicate that anticholinergic agents aggravate psychotic symptoms and antagonize therapeutic effects of neuroleptics in schizophrenic patients; more recently, some investigators have observed that these agents appear to benefit negative symptoms. In an effort to resolve this issue, we studied the effects of $\mathbf{2}$ days of treatment with biperiden on positive and negative symptoms in 15 medication-free schizophrenic patients. Positive symptoms increased significantly, while there was a trend toward a decrease in negative symptoms. The implications of these findings for the role of the cholinergic system in schizophrenia are discussed.
\end{abstract}

Key Words. Biperiden, cholinergic system, schizophrenia, positive symptoms.

Anticholinergic drugs are commonly used in the treatment of extrapyramidal side effects, which frequently accompany neuroleptic treatment in schizophrenia. It is assumed that anticholinergic drugs do not affect schizophrenic symptomatology, and general clinical experience is consistent with this assumption. There were some early reports, however, of anticholinergic drugs exacerbating psychotic symptomatology in schizophrenic patients (Gershon and Olariu, 1960; Itil et al., 1969). These observations have been supported by several recent studies showing that antimuscarinic/anticholinergic agents may antagonize the therapeutic effects of neuroleptics in schizophrenia (Singh and Kay, 1979; Johnstone et al., 1983, 1988; Singh et al., 1987). The adverse effects of anticholinergic drugs in schizophrenia are reportedly confined to positive symptoms, without any effects on negative symptoms. In fact, some recent studies indicate that trihexiphenidyl, an antimuscarinic agent, may actually have beneficial effects on negative schizophrenic symptoms (Fayen et al., 1988; Tandon et al., 1988).

In view of the frequent use of anticholinergic agents in schizophrenia, the dearth of controlled studies on the effects of anticholinergic drugs on symptomatology in medication-free schizophrenic patients is surprising. In this study, we examined the effects of biperiden, a relatively specific $M_{1}$ antimuscarinic/anticholinergic agent, on

Rajiv Tandon, M.D., is Director, Schizophrenia Program, and Assistant Professor, Department of Psychiatry, University of Michigan Medical Center, Ann Arbor, MI. Nancy A. Mann, R.N., William H. Eisner, R.N., and Nancy Coppard, R.N., are in the Schizophrenia Research Program and Department of Psychiatric Nursing, University of Michigan, Ann Arbor, MI. (Reprint requests to Dr. R. Tandon, UH-9C-9150, University of Michigan Medical Center, Ann Arbor, MI 48109-0120, USA.) 
positive and negative symptoms in 15 schizophrenic inpatients who were otherwise free of medication.

\section{Methods}

Patients and Study Design. The sample consisted of 15 consecutively hospitalized patients who were admitted to the Schizophrenia Inpatient Program at the University of Michigan. Diagnostic evaluation included a structured interview using the Schedule for Affective Disorders and Schizophrenia (SADS; Endicott and Spitzer, 1978), as well as all available history and clinical observations. Patients had to meet both $D S M-I I I-R$ criteria (American Psychiatric Association, 1987) and Research Diagnostic Criteria (RDC; Spitzer et al., 1978) for inclusion in the study. The sample consisted of 11 men and 4 women with a mean age of $29(S D=6)$ years and a mean duration of illness of $6(\mathrm{SD}=4)$ years. All of the patients were free of medical disorders. None of them had received depot neuroleptics for at least 6 months before entering the study.

After having been maintained in a medication-free state for at least 2 weeks, patients received $4 \mathrm{mg}$ of biperiden on night 1 , two doses of $4 \mathrm{mg}$ biperiden the next day, and a $4 \mathrm{mg}$ morning dose of biperiden on day 3. Two nurse-clinician raters (N.A.M. and W.H.E.), without knowledge of patients' medication status and the rationale of the study, independently rated the patients on the 18-item Brief Psychiatric Rating Scale (BPRS; Overall and Gorham, 1962) before starting biperiden on day 1 (baseline) and day 3 (postbiperiden). Scores on BPRS items assessing motor retardation, blunted affect, and emotional withdrawal (rated 1 for not present to 7 for severe) were summed and used as the BPRS negative symptom cluster. These items collectively constitute the "ANER" factor (Guy, 1976; Hedlund and Viewig, 1980). The BPRS items assessing conceptual disorganization, suspiciousness, hallucinatory behavior, and unusual thought content were summed as the BPRS positive symptom cluster. The BPRS was used to rate positive and negative symptomatology because it is a reliable, sensitive, and effective measure of psychopathology and of treatment-related symptom changes (Hedlund and Viewig, 1980).

Biperiden was used as the antimuscarinic/anticholinergic agent because, as a relatively specific $M_{1}$ antimuscarinic antagonist (Syvalähti et al., 1987; Eltze and Figala, 1988), it causes significantly fewer peripheral anticholinergic side effects than other commonly used oral anticholinergic agents and is better tolerated (Avissar and Schreiber, 1989). Furthermore, $M_{1}$ receptors are located in the brain areas considered relevant in schizophrenia (Bonner et al., 1987; Watson et al., 1987) and are thus the receptor system most likely to be implicated in schizophrenic symptomatology. Since the usual daily dosage of biperiden in the treatment of parkinsonian symptoms is $4-12 \mathrm{mg} /$ day, we elected to use $8 \mathrm{mg}$ in two divided doses. The 3-day duration of the study was selected because (1) the effect of a single dose on symptomatology may be limited by the inability of the system to respond this quickly to anticholinergic modulation; and (2) compensatory changes to the initial primary effect of biperiden might occur over a longer period, thereby interfering with the evaluation of the primary effect.

Data Analysis. Paired two-tailed $t$ tests were performed to compare the symptom ratings at baseline to those in the postbiperiden phase.

\section{Results}

The BPRS total scores at baseline ranged from 35 to 72 (mean $=48.2, \mathrm{SD}=12.3$ ). In this study, interrater reliability coefficients (measured by the Pearson productmoment correlations) between the two independent raters (N.A.M. and W.H.E.) for the 18 individual BPRS items ranged from 0.70 to 0.95 , with a median of 0.85 . The interrater reliability coefficients for the positive and negative symptom clusters were 0.92 and 0.87 , respectively.

Baseline positive symptom cluster scores ranged from 7 to 20 (mean $=12.8, \mathrm{SD}=$ 
3.6). There was a significant increase in positive symptom severity following biperiden $(t=4.4, d f=14, p<0.01)$, with postbiperiden positive symptom cluster scores ranging from 6 to 22 (mean $=15.7, \mathrm{SD}=4.4$ ). (See Fig. 1.)

Baseline negative symptom cluster scores ranged from 3.5 to 16.5 (mean $=9.0, \mathrm{SD}=$ 3.7). There was a trend toward a decrease in negative symptom severity following biperiden $(t=-1.9, d f=14, p=0.08)$, with postbiperiden negative symptom cluster scores ranging from 4 to 14 (mean $=7.9, \mathrm{SD}=3.0$ ). (See Fig. 1.) There was no association between changes in positive and negative symptom severity $(r=0.27, n=$ 15, NS).

Daily monitoring of patients' clinical status by their respective primary nurses (different from the nurse-clinician raters) was consistent with the changes in positive and negative symptoms noted on the BPRS. After biperiden, patients were reported as being more "open," expressive, engaging, and communicative. The primary nurses also noted an increased reporting of positive symptomatology (e.g., delusions and hallucinations) by the patients, which they ascribed to their increased "openness" and expressiveness.

Fig. 1. Change in positive and negative symptoms following biperiden in schizophrenic patients $(n=15)$
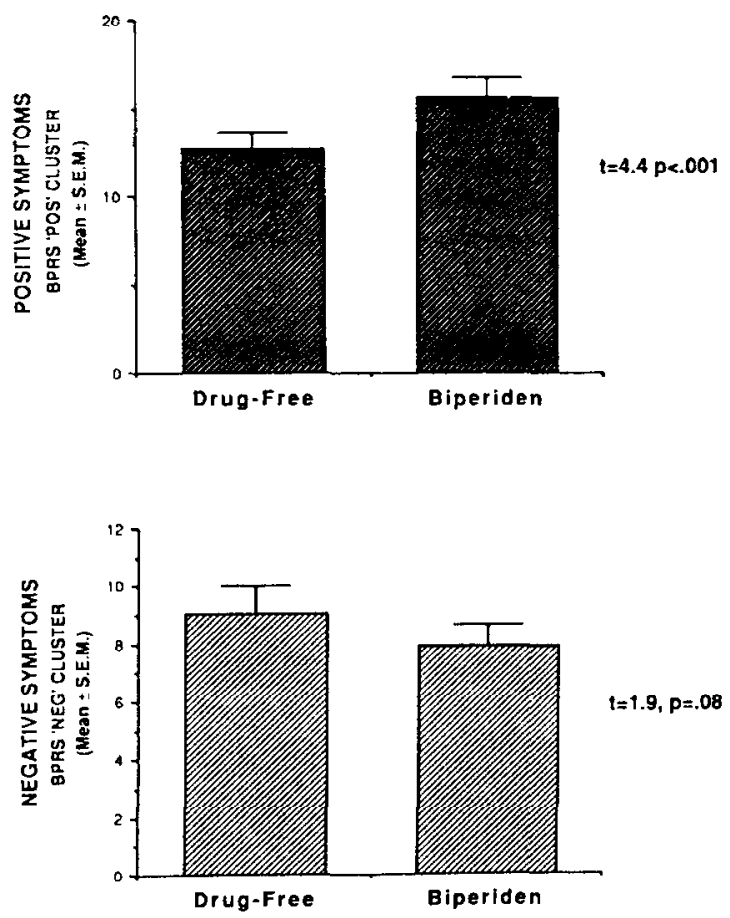


\section{Discussion}

The finding that the severity of positive symptoms increases following the administration of biperiden to medication-free schizophrenic patients is consistent with previous reports of aggravation of psychotic symptomatology (Gershon and Olariu, 1960; Itil et al., 1969) and antagonism of the therapeutic effects of neuroleptics on positive symptoms (Haase, 1965; Singh and Kay, 1979; Johnstone et al., 1983, 1988; Singh et al., 1987) in schizophrenic patients by anticholinergic agents. On the other hand, several studies have demonstrated that abrupt or gradual withdrawal of anticholinergic drugs has no salutary effect on schizophrenic symptoms and, in fact, may occasionally be associated with a worsening of the clinical state (Ananth et al., 1970; McClelland et al., 1974; Rifkin et al., 1975; Jellinek et al., 1981; Manos et al., 1981). A review of the literature reveals that studies showing no adverse effects of anticholinergic drugs and no improvement following anticholinergic withdrawal in schizophrenic patients were conducted in the chronic neuroleptic-stabilized phase of the illness, while investigations documenting adverse effects of anticholinergic drugs on positive symptoms were conducted in the acute phase of the illness. In any event, virtually all these studies were conducted in schizophrenic patients receiving neuroleptics, and any effect of reducing cholinergic activity could have been obscured by the simultaneously decreased dopaminergic activity due to the neuroleptic. In the present study, the effects of biperiden were evaluated in otherwise medication-free patients, thereby eliminating the "confounding" effect of neuroleptic medication. The importance of disturbances in dopaminergic/cholinergic balance in the expression of positive schizophrenic symptoms is supported by the finding that the exacerbation in positive symptoms produced by methylphenidate (an agent that enhances dopamine neurotransmission) in schizophrenic patients is reversed by physostigmine (an agent that enhances cholinergic neurotransmission) (Janowsky et al., 1973).

While positive symptoms increased following biperiden, negative symptoms showed a tendency to decrease. The observed reduction in negative symptoms with biperiden is consistent with previous reports of beneficial effects of anticholinergic agents on negative schizophrenic symptoms (Fayen et al., 1988; Tandon et al., 1988). In these earlier studies, however, patients were also receiving neuroleptic drugs, raising the possibility that the beneficial effects of the anticholinergic agents were related to relief of akinesia, despite the absence of any overt extrapyramidal side effects. The present study, in which patients were medication free except for the "study drug" biperiden, makes that interpretation less likely.

In view of the covariance of positive and negative symptoms in the psychotic phase of schizophrenia and their concomitant response to neuroleptic treatment (Goldberg, 1985; Breier et al., 1987; van Kammen et al., 1987; Goldman and Tandon, 1989; Kay and Singh, 1989; Keefe et al., 1989), our findings of an increase in positive symptoms and a decrease in negative symptoms following a reduction in cholinergic activity (with biperiden) appear puzzling. They are, however, consistent with and predictable from the recently proposed model of dopaminergic/cholinergic interactions in schizophrenia (Tandon and Greden, 1989, in press). This model suggests that (1) cholinergicdopaminergic balance is of central importance in schizophrenic pathophysiology; (2) muscarinic activity increases in an attempt to maintain this balance in the face of 
increasing dopaminergic activity that occurs in the psychotic phase of the illness; (3) the muscarinic/cholinergic system exerts a damping effect on the emergence of positive symptoms associated with dopaminergic hyperactivity; and (4) this compensatory incrcase in muscarinic activity is in turn accompanied by an intensification of negative symptoms during and following the psychotic phase of the illness.

Reducing cholinergic activity without concomitantly decreasing dopaminergic activity (as in the present study) would be expected to increase positive symptoms, as the cholinergic system may afford protection against the emergence of positive symptoms associated with dopaminergic hyperactivity. At the same time, decreasing cholinergic activity would be expected to result in a reduction of negative symptoms (as the "compensatory" cholinergic hyperactivity is implicated as a major mechanism in the production of negative symptoms at this phase of the illness). On the other hand, both positive and negative symptoms would be expected to improve with neuroleptic treatment. As dopaminergic activity decreases with neuroleptic treatment (and positive symptoms improve), there should be a corresponding reduction in muscarinic activity, since an increase in muscarinic activity presumably occurred as a "compensatory" response to the increased dopaminergic activity in this phase of the illness. This reduction in muscarinic/cholinergic activity would be accompanied by an improvement in negative symptoms.

Several methodological issues deserve comment: (1) Anticholinergic drugs, particularly benztropine, may cause dopamine release and block reuptake (thereby increasing central dopaminergic activity) (Horn et al., 1971; Modell et al, 1989). Thus, the increase in positive symptoms and the decrease in negative symptoms with biperiden could possibly be ascribed to its dopaminergic rather than its anticholinergic property. Commonly used anticholinergic drugs other than benztropine, however, have very weak effects on the dopamine and other catecholamine receptors, and biperiden has the least potent activity in this regard (Horn et al., 1971). While it is therefore unlikely that the observed effects of biperiden on positive and negative symptoms are related to its insignificant dopaminergic activity, this alternative explanation cannot be completely dismissed. (2) While the increase in positive symptoms that occurred with biperiden was very highly significant, the decrease in negative symptoms only showed a trend toward significance $(p=0.08)$. Whether this reflects the need for a larger sample, limitations of our instruments, or absence of a real effect needs to be evaluated in future studies. Even if these effects of an anticholinergic agent on positive and negative symptoms were confirmed, the clinical relevance of such findings would require further evaluation. (3) These findings must be considered preliminary and require corroboration in larger samples with a placebo-controlled design.

If these findings are confirmed, they have important pathophysiological implications for the role of the cholinergic system in schizophrenia. They may also have implications for therapy in view of (1) the widespread use of anticholinergic agents in the treatment of extrapyramidal side effects of neuroleptics and (2) the fact that many neuroleptic drugs also have potent anticholinergic effects.

Acknowledgments. This research was supported by grants from the Biomedical Research Council and the Department of Psychiatry, University of Michigan. 


\section{References}

American Psychiatric Association. DSM-III-R: Diagnostic and Statistical Manual of Mental Disorders. 3rd ed., revised. Washington, DC: American Psychiatric Press, 1987.

Ananth, J.V.; Horodesky, J.; Lehmann, H.E.; and Ban, T.A. Effect of withdrawal of antiparkinsonian medication on chronically hospitalized psychiatric patients. Laval Medicale, 41:934-938, 1970.

Avissar, S., and Schreiber, G. Muscarinic receptor subclassification and G-proteins: Significance for lithium action in affective disorders and for the treatment of extrapyramidal sideeffects of neuroleptics. Biological Psychiatry, 26:113-130, 1989.

Bonner, T.I.; Buckley, N.J.; Young, A.C.; and Brann, M.R. Identification of a family of muscarinic acetylcholine receptor genes. Science, 237:527-532, 1987.

Breier, A.; Wolkowitz, O.M.; Doran, A.R.; Roy, A.; Boronow, J.; Hommer, D.W.; and Pickar, D. Neuroleptic responsivity of negative and positive symptoms in schizophrenia. American Journal of Psychiatry, 144:1549-1555, 1987.

Eltze, M., and Figala V. Affinity and selectivity of biperiden enantiomers for muscarinic receptor subtypes. European Journal of Pharmacology, 158:11-19, 1988.

Endicott, J., and Spitzer, R.L. A diagnostic interview: The Schedule for Affective Disorders and Schizophrenia. Archives of General Psychiatry, 35:837-853, 1978.

Fayen, M.; Goldman, M.B.; Moulthrop, M.A.; and Luchins, D.J. Differential memory function with dopaminergic versus anticholinergic treatment of drug-induced extrapyramidal symptoms. American Journal of Psychiatry, 145:483-486, 1988.

Gershon, S.. and Olariu, J. JB 329-A new psychotomimetic: Its antagonism by tetrahydroaminacrin and its comparison with LSD, mescaline, and sernyl. Journal of Neuropsychiatry, 1:283-292, 1960.

Goldberg, S.C. Negative and deficit symptoms do respond to neuroleptics. Schizophrenia Bulletin, 11:453-456, 1985.

Goldman, R., and Tandon, R. Mutability and relationship between positive and negative symptoms during neuroleptic treatment in schizophrenia. Biological Psychiatry, 25:103-104A, 1989.

Guy, W. ECDEU Assessment Manual for Psychopharmacology. (DHEW Publication No.76-338) Rockville, MD: National Institute of Mental Health, 1976.

Haase, H.-J. The relationship of neuroleptic action to extrapyramidal phenomena. In: Haase, H.-J., and Janssen, P.A.J., eds. The Action of Neuroleptic Drugs. Amsterdam: NorthHolland Publishing Company, 1965. pp. 56-103.

Hedlund, J.L., and Viewig, B.W. The Brief Psychiatric Rating Scale (BPRS): A comprehensive review. Journal of Operational Psychiatry, 11:48-65, 1980.

Horn, A.S.; Coyle, J.T.; and Snyder, S.H. Catecholamine uptake by synaptosomes from rat brain: Structure-activity relationship of drugs with differential effects on dopamine and norepinephrine neurons. Molecular Pharmacology, 7:66-80, 1971.

Itil, T.M.; Keskiner, A.; and Holden, J.M.C. The use of LSD and ditran in the treatment of therapy-resistant schizophrenics. Diseases of the Nervous System, 30:93-103, 1969.

Janowsky, D.S.; El-Yousef, M.K.; Davis, J.M.; and Sekerke, H.J. Antagonistic effects of physostigmine and methylphenidate in man. American Journal of Psychiatry, 130:1370-1376, 1973.

Jellinek, T.; Gardos, G.; and Cole, J.O. Adverse effects of antiparkinson drug withdrawal. American Journal of Psychiatry, 138:1567-1571, 1981.

Johnstone, E.C.; Crow, T.J.; Frith, C.D.; and Owens, D.G.C. Adverse effects of anticholinergic medication on positive schizophrenic symptoms. Psychological Medicine, 13:513$527,1983$.

Johnstone, E.C.; Crow, T.J.; Frith, C.D.; and Owens, D.G.C. The Northwick Park "Functional" Psychosis Study: Diagnosis and treatment response. Lancet, II:119-125, 1988.

Kay, S.R., and Singh, M.M. The positive-negative distinction in drug-free schizophrenic patients. Archives of General Psychiatry, 46:711-718, 1989. 
Keefe, R.S.E.; Harvey, P.D.; Bergman, R.L.; Mohs, R.C.; and Davis, K.L. State and trait components of negative symptoms within schizophrenic patients. Biological Psychiatry, 25:105A, 1989.

Manos, N.; Gkiouzepas, J.; Tzotzaras, T.; and Tzanetoglou, A. Gradual withdrawal of antiparkinson medication in chronic schizophrenics: Any better than the abrupt? Journal of Nervous and Mental Disease, 169:659-661, 1981.

McClelland, H.A.; Blessed, G.; Bhate, S.; Ali, N.; and Clarke, P.A. The withdrawal of antiparkinsonian drugs in schizophrenia patients. British Journal of Psychiatry, 124:151-159, 1974.

Modell, J.G.; Tandon, R.; and Beresford, T. Dopaminergic activity of the "antimuscarinic" antiparkinsonian agents. Journal of Clinical Psychopharmacology, 9:347-351, 1989.

Overall, J.E., and Gorham, D.R. The Brief Psychiatric Rating Scale. Psychological Reports, 10:799-812, 1962.

Rifkin, A.; Quitkin, F.; and Klein, D. Akinesia: A poorly recognized drug-induced extrapyramidal behavioral disorder. Archives of General Psychiatry, 32:672-674, 1975.

Singh, M.M., and Kay, S.R. Therapeutic antagonism between anticholinergic antiParkinsonism agents and neuroleptics in schizophrenia: Implications for a neuropharmacological model. Neuropsychobiology, 5:74-86, 1979.

Singh, M.M.; Kay, S.R.; and Opler, L.A. Anticholinergic-neuroleptic antagonism in terms of positive and negative symptoms in schizophrenia: Implications for psychobiological typing. Psychological Medicine, 17:39-48, 1987.

Spitzer, R.L.; Endicott, J.; and Robins, E. Research Diagnostic Criteria: Rationale and reliability. Archives of General Psychiatry, 35:773-782, 1978.

Syvalähti, E.K.G.; Lauren, L.; Markannen, J.; and Kunelius, R. Interaction of psychotropic drugs with brain muscarinic cholinoceptors: Similarities of biperiden and pirenzipine in receptor binding properties. Pharmacology and Toxicology, 60:66-69, 1987.

Tandon, R., and Greden, J.F. Cholinergic hyperactivity and negative schizophrenic symptoms: A model of cholinergic/dopaminergic interactions in schizophrenia. Archives of General Psychiatry, 46:745-753, 1989.

Tandon, R., and Greden, J.F. Cholinergic excess and negative symptoms. In: Greden, J.F., and Tandon, R., eds. Negative Schizophrenic Symptoms: Pathophysiology and Clinical Implications. Washington, DC: American Psychiatric Press, in press.

Tandon, R.; Greden, J.F.; and Silk, K.R. Treatment of negative schizophrenic symptoms with trihexiphenidyl. Journal of Clinical Psychopharmacology, 8:212-215, 1988.

van Kammen, D.P.; Hommer, D.W.; and Malas, K.L. Effect of pimozide on positive and negative symptoms in schizophrenic patients: Are negative symptoms state dependent? Neuropsychobiology, 18:113-117, 1987.

Watson, M.; Roeske, W.R.; and Yamamura, H.I. Cholinergic receptor heterogeneity. In: Meltzer, H.Y., ed. Psychopharmacology: The Third Generation of Progress. New York: Raven Press, 1987. pp. 241-248. 